\title{
Efficacy and Safety of Remimazolam Tosilate versus Propofol for General Anesthesia in Cirrhotic Patients Undergoing Endoscopic Variceal Ligation
}

Fu Shi

Yanjie Chen

Hongtao Li

Yang Zhang

Tonghang Zhao

Department of Anesthesiology, Liaocheng People's Hospital, Liaocheng,

252000, People's Republic of China
Correspondence: Tonghang Zhao

Email zthlymz@163.com
Introduction: The best candidate intravenous anesthetic agent for patients with liver cirrhosis undergoing endoscopic variceal ligation (EVL) remains unclear. Remimazolam tosilate (RT) is a new type of benzodiazepine with quick onset, rapid recovery, and no accumulation. Here, we investigated the efficacy and safety of RT for general anesthesia in cirrhotic patients undergoing EVL.

Methods: Patients undergoing EVL were randomly classified into the remimazolam tosilate (group R) and the propofol group (group P). RT was administered as a slow bolus of $0.2 \mathrm{mg} /$ $\mathrm{kg}$ for induction and followed by $1.0-2.0 \mathrm{mg} / \mathrm{kg} / \mathrm{h}$ for maintenance of general anesthesia. Propofol was started at $2 \mathrm{mg} / \mathrm{kg}$, followed by $4-10 \mathrm{mg} / \mathrm{kg} / \mathrm{h}$ until the end of surgery. Flumazenil was routinely administered to group $\mathrm{R}$ and the same volume of saline was given to group P immediately after surgery. The efficacy and safety of RT for general anesthesia during EVL were compared with propofol.

Results: All patients in the two groups had satisfactory anesthetic effects and the efficacy rates were $100 \%$. The time to loss of consciousness (LoC) was longer in group $\mathrm{R}$ than in group $\mathrm{P}(P>0.05)$. The return of consciousness $(\mathrm{RoC})$ time, extubation time, and transfer time in group $\mathrm{R}$ were significantly shorter than that in group $\mathrm{P}(P<0.05)$. The incidence of intraoperative hypotension and postoperative low $\mathrm{SpO}_{2}$ in group $\mathrm{R}$ were lower than that in the group $\mathrm{P}(P<0.05)$. There were no significant differences between the two groups with respect to the satisfaction degree of patients and operators $(P>0.05)$.

Conclusion: Remimazolam tosilate can provide satisfactory anesthetic effects for surgery. Group R patients recovered faster and had a shorter PACU stay time than group P patients. Moreover, RT decreased the incidence of hypotension and low $\mathrm{SpO}_{2}$. RT was a safer and more effective alternative for general anesthesia in cirrhotic patients undergoing EVL than propofol.

Keywords: remimazolam tosilate, propofol, general anesthesia, endoscopic variceal ligation, liver cirrhosis

\section{Introduction}

Esophageal and gastric varices bleeding (EGVB) is one of the common serious complications in patients with cirrhosis and the mortality rate is up to $20 \%{ }^{1}$ Endoscopic variceal ligation (EVL) has become the favored treatment and prophylaxis for EGVB. ${ }^{2}$ With the increase of outpatient EVL surgeries, how to ensure an anesthetic effect and safety of these patients has become one of the clinical focuses.

Intravenous anesthesia can improve the tolerance and cooperation of patients and provide a stable operating environment for endoscopists. The most popular intravenous agents for cirrhotic patients are midazolam and propofol that are both 
equally acceptable by patients and operators. ${ }^{3}$ Nevertheless, liver cirrhosis can damage protein synthesis, change drug metabolic pathways, and damage liver blood flow, and all these factors affect the pharmacokinetics of anesthetic agents. ${ }^{4}$ Both midazolam and propofol that are used for cirrhotic patients have advantages and disadvantages. Midazolam has rapid action and anterograde amnesia properties and is widely used in gastroenterological endoscopy. ${ }^{5}$ However, midazolam eliminates half-life is up to 1.8 to $6.4 \mathrm{~h}$ and the active metabolites can cause delays in neuropsychiatric recovery, especially in patients with renal or liver dysfunction or those receiving protease inhibitors. ${ }^{6,7}$ One study showed that midazolam used for patients with cirrhosis increased the risk of hepatic encephalopathy. ${ }^{8}$ Propofol is the most preferred anesthetic agent owing to its quick effect, short half-life, and rapid recovery properties. It also reported that propofol can be safer and more effective than midazolam in cirrhosis patients undergoing upper GI endoscopy. ${ }^{9}$ However, subjects with hepatic impairment showed increased sensitivity to cardiopulmonary inhibitory effects of propofol and surgical stress. ${ }^{10,11}$ Furthermore, propofol has a narrow safety range that may carry potential risks including hypoxemia, hypotension, and bradycardia during endoscopic procedures, especially in patients with cirrhosis. ${ }^{12}$ With respect to safety, it requires fast induction and revival, perfect depth of anesthesia, stable hemodynamics, and no obvious residual effect. Therefore, reasonable choice and proper application of anesthetic agents are essential for cirrhotic patients.

Remimazolam tosilate (RT) (developed by Jiangsu HengRui Medicine Co. Ltd., China) is a new type of benzodiazepine that activates $\mathrm{GABA}_{\mathrm{A}}$ receptor producing a sedative effect. RT is hydrolyzed by tissue esterase to inactive metabolites and can be rapidly antagonized by flumazenil. ${ }^{13}$ RT has been used for outpatients in upper gastrointestinal endoscopy, which can reach the same depth of anesthesia as propofol and show faster recovery than propofol. ${ }^{14}$ To our knowledge, no explicit recommendation has yet been made on the safety of RT for EVL under general anesthesia for patients with cirrhosis. Furthermore, it is unclear whether RT can provide the same benefits for cirrhotic patients. Therefore, this clinical trial was first undertaken to evaluate the efficacy and safety of RT versus propofol for general anesthesia in Chinese patients with cirrhosis undergoing EVL.

\section{Methods-Study Design}

The present study was approved by the Ethics Committee of the Liaocheng People's Hospital in Shandong Province, China. The study was carried out according to the guidelines of the Declaration of Helsinki and registered in the Chinese Clinical Trial Registry on April 23, 2021 (ChiCTR2100045710; main researcher: Fu Shi). This study was a prospective, single-center, randomized, singleblind, controlled trial comparing RT and propofol (AstraZeneca, USA) for parallel trials of general anesthesia in cirrhotic patients undergoing endoscopic variceal ligation (EVL). All recruited patients signed the informed consent form. This study was carried out between April 26, 2021 and September 18, 2021. A total of 76 patients with American Society of Anesthesiologists (ASA) physical status II or III, who were consecutively scheduled undergoing EVL at the Endoscopy Examination Center of the Liaocheng People's Hospital were enrolled in the trial. They were randomly divided into two groups by using a computer to generate a random number list at a 1:1 ratio ( $\mathrm{n}=38$ patients in each group). We used a single-blind design because of the difference of drug color and dosage form. The random group sequence number was placed in an opaque envelope by a nurse. Another anesthesiologist opened the envelope and was aware of the treatment allocation of each patient. Neither the participants nor the outcome evaluators were aware of the treatment allocation throughout the study.

The inclusion criteria were patients between 20 and 80 years of age; patients with a recent history of bleeding who planned to receive surgery; and those who signed the informed consent. The exclusion criteria were ASA IV or $\mathrm{V}$; acute active variceal bleeding; alcohol consumption in the past 2 weeks; hepatic encephalopathy; neurological diseases such as Alzheimer's or Parkinson's disease; peripheral hemorrhagic shock; allergy to benzodiazepine or propofol; and refusal to sign the informed consent.

\section{Operative Procedure}

All operations were performed by experienced hepatologists with more than 5 years of EVL experience. A standard endoscope (GIF-H260, outer diameter of $9.8 \mathrm{~mm}$; Olympus Optical Co., Ltd., Tokyo, Japan) was used for recording the gastric bottom varicose veins and determining the parts to be ligated. Varicose veins were fully attracted to "red" in the transparent cap, then circles 
were released using ligation device (Six Shooter; Cook Medical, Winston-Salem, NC, USA) esophageal.

\section{Anesthesia and Intervention Induction of General Anesthesia}

Before surgery, an anesthetist explained the anesthesia risk to patients and obtained their written consent for anesthesia induction. In the operating room, the patient was placed in a lateral position, and a venous channel was established on the right upper limb; no invasive upper pressure was detected in the left upper limb. Monitoring vital signs included electrocardiogram (ECG), heart rate (HR), mean arterial blood pressure (MAP), pulse oxygen saturation $\left(\mathrm{SpO}_{2}\right)$, end-tidal $\mathrm{CO}_{2}$ partial pressure $\left(\mathrm{PetCO}_{2}\right)$ and bispectral index (BIS). The depth of anesthesia was evaluated by the Modified Observer's Assessment of Alertness/Sedation (MOAA/S: 5 points, responsive to normal verbal stimuli sensitively; 4 points, responsive to normal verbal stimuli bluntly; 3 points, responsive to loud or repeated verbal stimuli; 2 points, responsive to a slight push and vibration; 1 point, responsive only after painful trapezius squeeze; and 0 , no response even to painful trapezius squeeze). ${ }^{15}$

In our study, we used the rapid sequence intubation that can effectively prevent the risk of perioperative reflux aspiration. Before induction of general anesthesia, $5 \mathrm{~L} / \mathrm{min}$ oxygen was given by a mask for $3 \mathrm{~min}$. At the same time, $3 \mu \mathrm{g} / \mathrm{kg}$ fentanyl citrate (Yichang Humanwell Pharmaceutical, China) was intravenously infused. In the study group (group R), general anesthesia induction for all patients was performed via a bolus of $0.2 \mathrm{mg} / \mathrm{kg} \mathrm{RT}$. In the control group (group P), a bolus of $2.0 \mathrm{mg} / \mathrm{kg}$ propofol was administered slowly. Loss of consciousness (LoC) was defined as a MOAA/S score $\leq 1$ in our study. After LoC was attained, endotracheal intubation was performed after $2 \mathrm{mg} / \mathrm{kg}$ intravenous succinylcholine.

\section{Maintenance of General Anesthesia}

Remimazolam tosilate was administered at $1 \mathrm{mg} / \mathrm{kg} / \mathrm{h}$ (maximum allowed infusion rate: $2 \mathrm{mg} / \mathrm{kg} / \mathrm{h}$ ) based on monitoring of the vital signs of individual subjects and MOAA/S scores during the surgery. Propofol was administered at 4 $10 \mathrm{mg} / \mathrm{kg} / \mathrm{h}$ based on monitoring of the vital signs of individual subjects and MOAA/S scores during the surgery. Remifentanil (Yichang Humanwell Pharmaceutical, China) was administered at an infusion rate $0.25 \mu \mathrm{g} / \mathrm{kg} / \mathrm{min}$. The infusion rate of drugs was adjusted as appropriate based on the MOAA/s score of each patient. The patients were sufficiently anesthetized with $\mathrm{MOAA} / \mathrm{s}=0$ for surgical operation.
It is still unclear whether RT and bispectral index (BIS) have a good correlation. The corresponding BIS indices that were equal to MOAA/s scores in our study were also recorded. The tidal volume was maintained at $6-8 \mathrm{~mL} / \mathrm{kg}$, and the respiratory frequency was maintained at 12 rates/min and end-tidal $\mathrm{CO}_{2}$ partial pressure was maintained at 35-45 $\mathrm{mmHg}$. Ephedrine $(0.1 \mathrm{mg} / \mathrm{kg}, \mathrm{IV})$ was administered if hypotension (systolic blood pressure descending 30\% basal value or $\mathrm{MAP}<65 \mathrm{mmHg}$ ) was detected. Intravenous atropine $(0.4 \mathrm{mg})$ was administered if bradycardia $(\mathrm{HR}<50 \mathrm{bpm})$ occurred.

\section{Recovery from General Anesthesia}

All the drugs were discontinued at the end of surgery. A bolus of $0.5 \mathrm{mg}$ flumazenil (Nhwa Pharma. Corporation, China) was administered to group $\mathrm{R}$ patients and the same volume of saline was given to group P. Return of consciousness (RoC) was defined as MOAA/S score $\geq 4$ in our study. The times to RoC and extubation were measured and recorded.

\section{Recovery from PACU}

After extubation, the patients were transferred to the postanesthesia care unit (PACU) for further observation. The investigator assessed and recorded the following: (i) adverse events, which included the prevalence of postoperative low $\mathrm{SpO}_{2}<95 \%$, and dizziness, nausea and vomiting (PONV), and intraoperative awareness after surgery; and (ii) transfer time (time taken from extubation to leaving the PACU).

\section{Clinical Outcomes}

The primary endpoint was the success rate of the surgical procedure. Successful criteria were defined as completion of the endoscopy procedure and deep sedation with MOAA/ $s=0$. Secondary efficacy endpoints included the following: (i) Time to $\mathrm{LoC}$, time to $\mathrm{RoC}$, time to extubation, and transfer time. (ii) Vital signs of patients especially mean arterial pressure (MAP) and heart rate (HR) during the surgery. The designated time points were as follows: before anesthesia induction (T0), after induction (T1), after intubation (T2), when surgery began (T3), 5 min after surgery (T4), 10 min after surgery (T5), 15 min after surgery (T6), $20 \mathrm{~min}$ after surgery (T7), end of surgery (T8), and $1 \mathrm{~min}$ after extubation (T9). (iii) The BIS index corresponding to $\mathrm{MOAA} / \mathrm{S}$ scores during general anesthesia. (iv) Incidence of hypotension during surgery and adverse events after surgery. (v) Patient and operator satisfaction, which were evaluated 
on a $0-10$ point visual analog scale, where 1 point indicated a very bad experience and 10 points indicated a perfect experience.

\section{Sample Size Calculation and Statistical Analysis}

Calculation of the sample size was undertaken by PASS 11.0 (NCSS, Kaysville, UT, USA). Based on the results of a pilot study involving 20 patients, the number of hypotension events during the surgery was $1.6 \pm 0.7$ in group $\mathrm{P}$ and $1.2 \pm 0.4$ in group R. Further, 31 patients in each group could achieve $80 \%$ power to detect a difference of 0.5 between the two groups. Considering a loss to followup of $20 \%, 38$ patients were required per group.

All experimental data were performed using SPSS software (version 17, IBM Corporation, Armonk, NY, USA), and $P<0.05$ was considered statistically significant. Data are expressed as the mean \pm standard deviation $(\overline{\mathrm{x}} \pm \mathrm{s})$ and were compared using the Student's $t$-test. Categorical variables are presented as percentage of numbers (\%) and were compared using a chi-square test. The GraphPad Prism software version 5.0 (GraphPad Prism, Inc., San Diego, CA, USA) was used for diagram drawing.

\section{Results}

A total of 88 patients were recruited for the study. Of these, three patients were in hemorrhagic shock, two patients had alcohol in the past 2 weeks, and five patients refused to sign the informed consent. Finally, 78 patients participated in the research. The data of two patients were lost in the process of research. Seventy-six patients $(n=38$ / group) completed the surgery (Figure 1). The characteristics of patients in the two groups are presented in Table 1. There were no significant differences between the groups in terms of age, sex, weight, height, and etiology of liver cirrhosis, ASA, and the duration of surgery $(P>0.05)$.

In both groups, all EVL surgeries were successfully performed (success rate: 100\%). As shown in Figure 2, the time to LoC in group $\mathrm{R}$ was longer than that in group $\mathrm{P}$ (group $\mathrm{R}$ : $45.7 \pm 5.3 \mathrm{~s}$; group R: $65.9 \pm 4.7 \mathrm{~s}, P>0.05)$. However, the time to $\mathrm{RoC}$ in group $\mathrm{R}$ was significantly shorter than that in group P (group P: $503.3 \pm 59.6$ s; group R: $67.1 \pm 9.6 \mathrm{~s}, P<0.05$ ). The time to extubation in group $\mathrm{R}$ was significantly shorter than that in group P (group P: $524.7 \pm 57.8 \mathrm{~s}$; group R: 115.7 \pm 12.5 $\mathrm{s}, P<0.05)$. The PACU stay time in group $\mathrm{R}$ was significantly shorter than that in group P (group P: $859.6 \pm 62.2$ s; group R: $370.2 \pm 24.1$ s, $P<0.05)$.

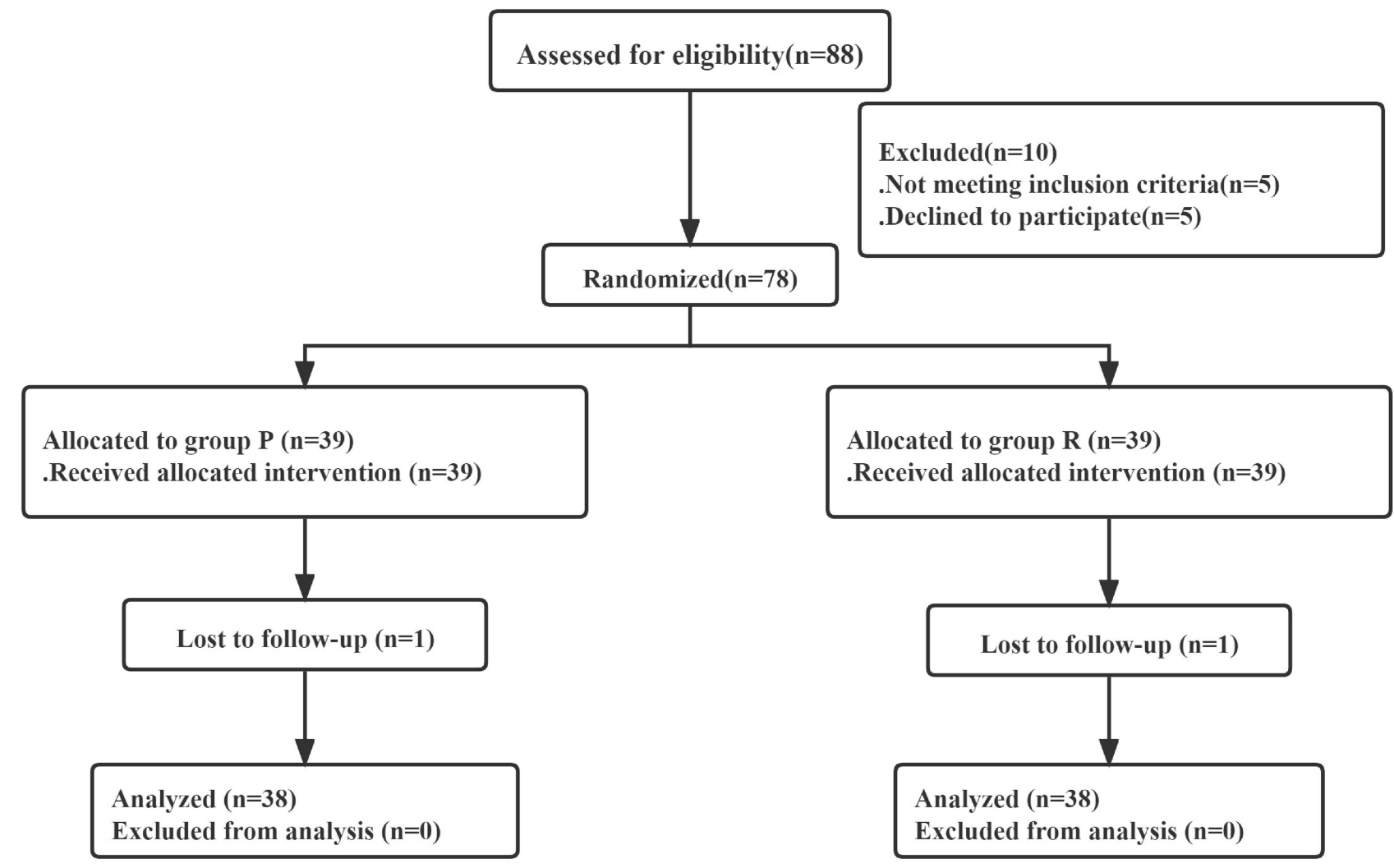

Figure I Study population flow diagram. 
Table I Patient Characteristics

\begin{tabular}{|l|l|l|l|}
\hline Variable & $\begin{array}{l}\text { Group } \\
\text { P (n=38) }\end{array}$ & $\begin{array}{l}\text { Group } \\
\text { R (n=38) }\end{array}$ & P value \\
\hline Age (years) & $51.61 \pm 5.48$ & $52.74 \pm 4.93$ & 0.347 \\
Sex (Male/Female) & $18 / 20$ & $16 / 22$ & 0.818 \\
Weight (kg) & $68.26 \pm 5.1 \mathrm{I}$ & $66.00 \pm 5.27$ & $0.06 \mathrm{I}$ \\
Height (cm) & $164.30 \pm 5.7 \mathrm{I}$ & $163.40 \pm 5.60$ & 0.493 \\
ASA, $\mathrm{n}(\%)$ & & & 0.637 \\
II & $22(57.9)$ & $25(65.8)$ & \\
III & $16(42.1)$ & $13(34.2)$ & \\
Etiology of liver cirrhosis, $\mathrm{n}(\%)$ & & & 0.754 \\
HBV & $31(81.6)$ & $33(86.8)$ & \\
Alcohol & $7(18.4)$ & $5(13.2)$ & \\
Duration of surgery (min) & $26.88 \pm 2.88$ & $27.00 \pm 2.72$ & 0.854 \\
\hline
\end{tabular}

Notes: Data are presented as mean \pm SD or numbers (\%). Group P, propofol group; Group R, RT group. $P<0.05$ was considered to indicate statistical significance.

The MAP and HR of 10 time points are summarized in the two groups, as shown in Figure 3. After anesthesia induction, the MAP at T1-8 in group P were significantly lower than that in group $\mathrm{R}(P<0.05)$. Compared with group $\mathrm{R}$, the $\mathrm{HR}$ at $\mathrm{T} 1$ and $\mathrm{T} 4-8$ in group $\mathrm{P}$ were all significantly reduced $(P<0.05)$, but all values at T1-8 were within the clinically normal range. Compared to group $\mathrm{P}$, group $\mathrm{R}$ showed less fluctuation in the MAP and HR.

In this study, we maintained the $\mathrm{MOAA} / \mathrm{s}=0$ after anesthesia induction (T1-T8) to ensure an adequately anesthetized state for surgical operation. No patient in group $\mathrm{R}$ required any other sedative drugs and could meet all surgical needs. After surgery, the MOAA/s score at $\mathrm{T} 9$ in group $\mathrm{R}$ was much higher than that in group $\mathrm{P}(P<0.05)$. We also detected BIS during the operation. As shown in Figure 4, the BIS scores at T1-T9 in group $\mathrm{P}$ were lower than that in group $\mathrm{R}(P<0.05)$.

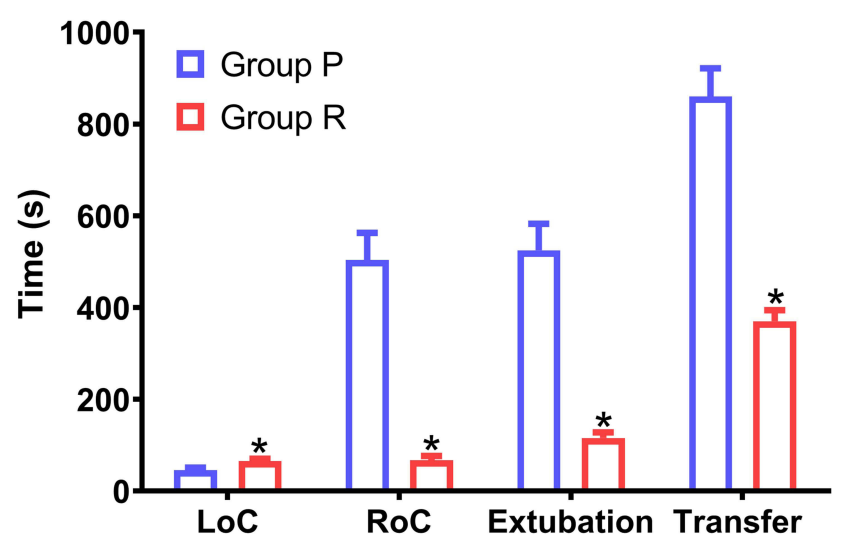

Figure 2 Induction and recovery time between the two groups. Transfer, PACU stay time. $* P<0.05$ was considered to indicate statistical significance.

Abbreviations: LoC, time to loss of consciousness; RoC, time to return of consciousness.
We compared the adverse events between the two groups (Figure 5). During anesthesia, the incidence of hypotension in group $\mathrm{P}$ was higher than that in group $\mathrm{R}(P<0.05)$. The incidence of post-operative low $\mathrm{SpO}_{2}$ in group $\mathrm{R}$ was significantly lower than that in group $\mathrm{P}(P<0.05)$. The incidence of other post-operative adverse events such as dizziness and PONV were similar in both groups $(P>0.05)$. Postoperative follow-up was performed for all patients, and no intraoperative awareness event occurred in the two groups.

The anesthesia satisfaction of patients and the conditions of surgery were assessed by a $0-10$ points visual analog scale (VAS) (Figure 6). The satisfaction score of patients and operator in group $\mathrm{R}$ were not significantly different from that in group $\mathrm{P}(P>0.05)$.

\section{Discussion}

To our knowledge, this study is the first to compare the efficacy and safety of RT versus propofol for general anesthesia in cirrhotic patients undergoing EVL. Based on our data, RT can provide satisfactory anesthetic effects for EVL and combination with flumazenil can induce quick recovery from anesthesia. Administration of flumazenil in combination with RT was a better alternative than propofol alone for general anesthesia during EVL.

In terms of cirrhotic patients undergoing EVL, the choice of anesthetic methods and agents present a huge challenge for the anesthesiologist. To the best of our knowledge, there are no specific anesthesia guidelines for EVL in cirrhotic patients. EVL in cirrhotic patients is mainly performed without sedation, under conscious or moderate sedation, and under general anesthesia. ${ }^{16-18}$ Unal and Saruc pointed out that EVL need high patient compliance and it may be technically more difficult without sedation. ${ }^{17}$ Gastroscopic irritation of the throat and esophagus can cause nausea, vomiting, choking, and agitation and it is easy to induce venous rupture and bleeding. The gastrointestinal bleeding may occur at any time during surgery. A previous study has shown that endotracheal intubation and general anesthesia are safe and effective for patients undergoing EVL. ${ }^{12}$ And, the airway can be ensured unobstructed with endotracheal intubation. In our study, no patient experienced aspiration during surgery, and no surgery was left incomplete owing to inadequate depth of anesthesia. So, endotracheal intubation under general anesthesia with RT or propofol may be of benefit for patient safety and procedural success. 

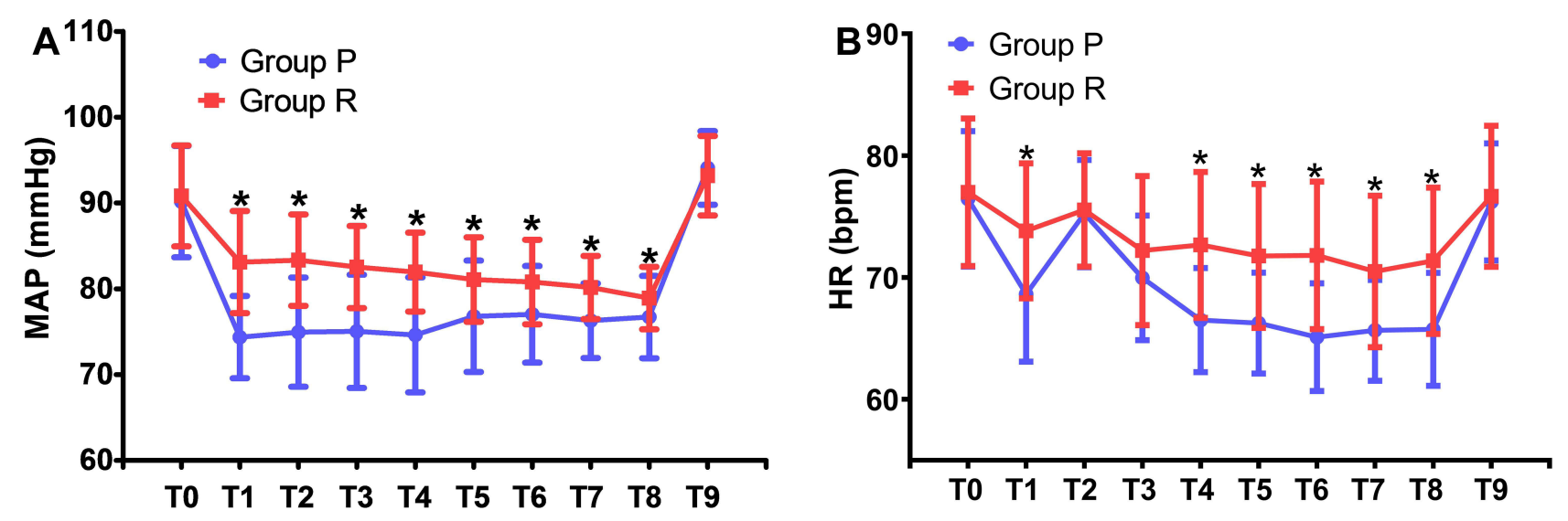

Figure 3 Intraoperative hemodynamic parameters in both groups (A and $\mathbf{B}$ ). ${ }^{*} P<0.05$ was considered to indicate statistical significance.

Abbreviations: MAP, mean arterial pressure; HR, heart rate.
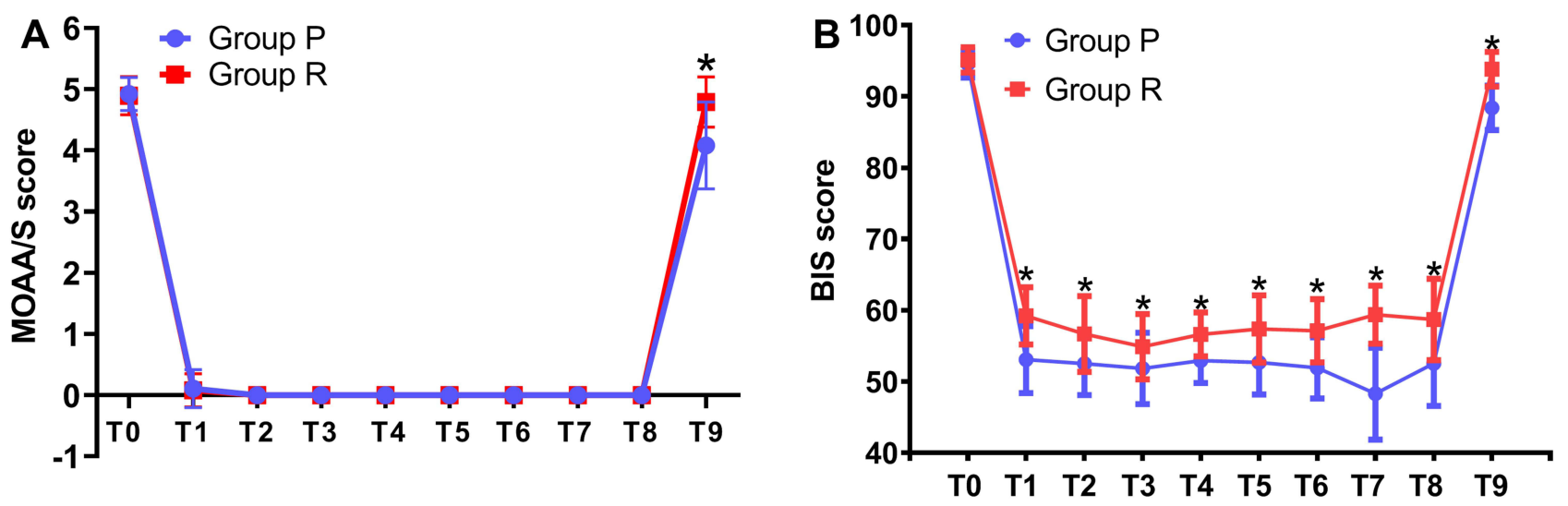

Figure 4 Intraoperative MOAA/s and BIS index scores in both groups (A and $\mathbf{B}$ ). $* P<0.05$ was considered to indicate statistical significance.

Benzodiazepines, opioids, and propofol were widely used for endoscopic procedures. ${ }^{19}$ Nevertheless, the halflife of midazolam was prolonged in cirrhotic patients, and midazolam sedation increased the incidence of adverse events especially for hepatic encephalopathy. ${ }^{16}$ Although propofol is superior to midazolam for sedation during endoscopic injection sclerotherapy, ${ }^{20}$ its pharmacological character in cirrhotic patients is still not well known. A basic study showed that rats with cirrhosis increased the efficacy of propofol anesthesia, and animals need longer recovery time, perhaps because of the reduced plasma proteins, increased inhibitory neurotransmitters, and decreased excitatory neurotransmitters. ${ }^{21}$ RT has a short elimination half-life without liver and kidney metabolism. ${ }^{22}$ A recent study showed that the pharmacokinetic properties of RT were not affected by hepatic impairment without dose adjustments. ${ }^{23}$ Moreover, flumazenil is a specific antagonist of RT. ${ }^{24}$ Our study showed that the mean times to $\mathrm{RoC}$ and extubation in group
$\mathrm{R}$ were significantly shorter than that in group P. Compared with propofol, RT significantly shortened the PACU stay time. However, a multicenter phase III trial found that the time to eye opening and extubation were statistically longer in the RT than the propofol group. ${ }^{25}$ In fact, we found that flumazenil was given to only $9.3 \%$ patients when we read the entire manuscript. The mean time to awakening in the RT group that received flumazenil was $1.8 \mathrm{~min}$. The reason for the difference may be that flumazenil was given for every patient in our study after surgery. In our study, the mean time to extubation was $115.7 \mathrm{~s}$, which was close to $1.8 \mathrm{~min}$. These statistically significant differences in our study had a major impact on the surgical procedures. Remimazolam tosilate plus sequential flumazenil enhanced the speed of recovery of cirrhotic patients and sped up the turnover of PACU beds. Regarding restoration of anesthesia, RT has a potential advantage over propofol and is also more controllable. 

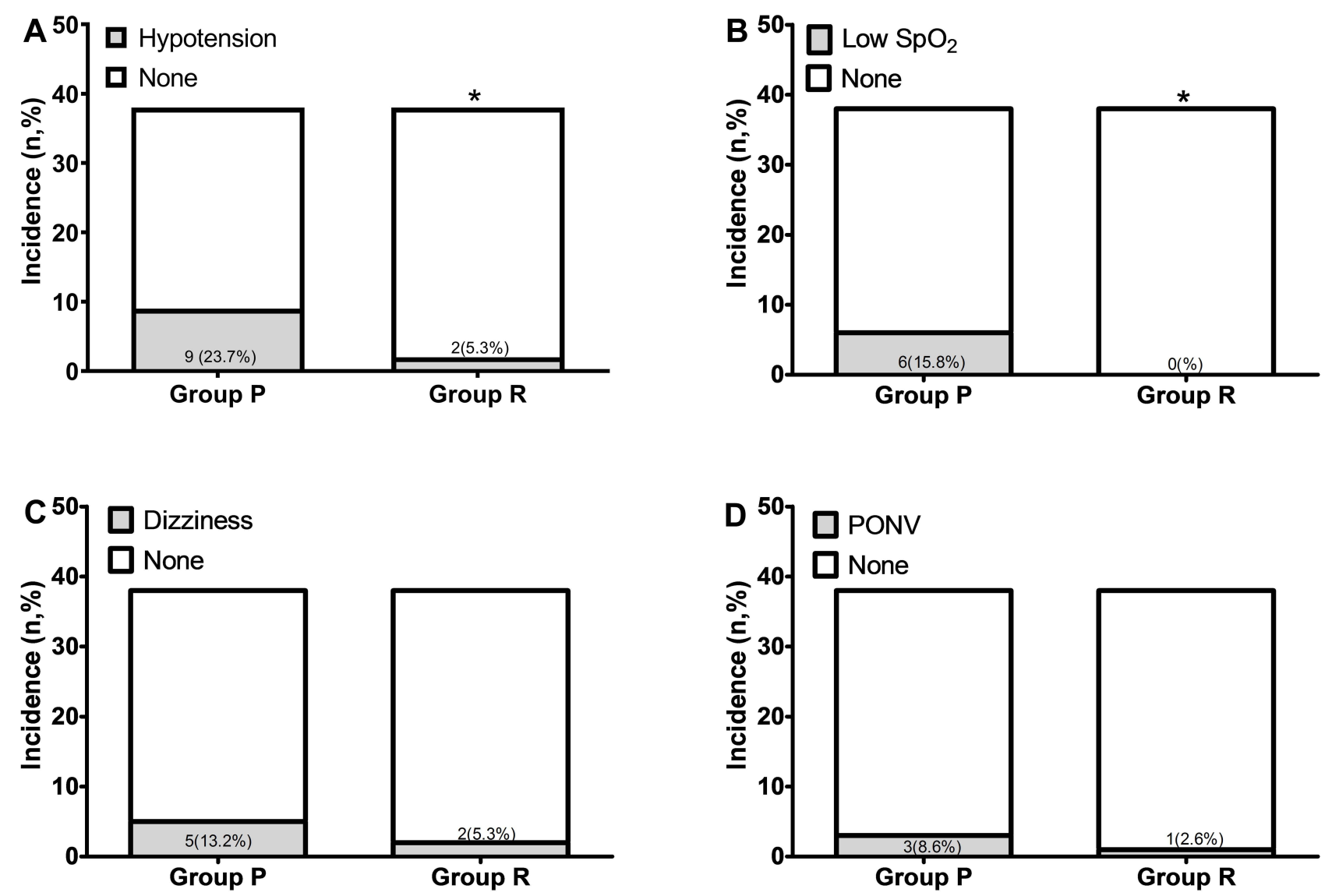

Figure 5 Comparisons of adverse events in both groups (A-D). $* P<0.05$ was considered to indicate statistical significance.

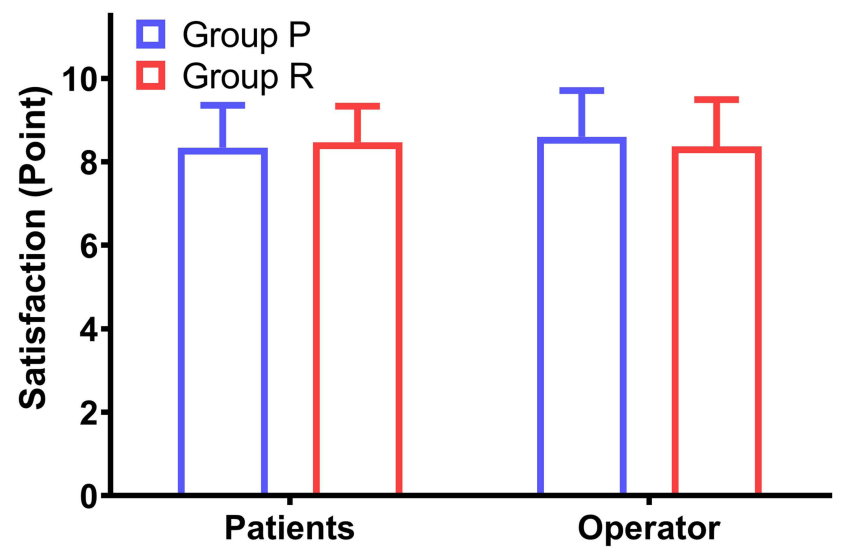

Figure 6 Satisfaction of patients and operator in the two groups after surgery. Satisfaction was assessed on a $0-10$ visual analog scale.

A recent clinical trial revealed that RT was equal to propofol with respect to efficacy for induction and maintenance of general anesthesia. ${ }^{25}$ Our study also showed that RT exhibited the same anesthetic performance with propofol anesthesia during surgery. The patients can achieve deep sedation with $\mathrm{MOAA} / \mathrm{s}=0$ under $\mathrm{RT}$ general anesthesia. The rate of success in the two groups was $100 \%$, and all cirrhotic patients could obtain sufficient anesthetic depth for surgery. BIS is the brain function monitor and the values between 45 and 60 have been recommended for general anesthesia. ${ }^{26}$ In our study, the mean BIS values under RT or propofol anesthesia were both within the adequate ranges of 4560. Furthermore, BIS scores during anesthesia in group $\mathrm{R}$ were significantly higher than those in group $\mathrm{P}(P<0.05)$. To a certain extent, RT avoided the deep anesthesia depth for patients. Compared with propofol anesthesia, the surgical operation conditions were not changed by using RT. The satisfaction scores of the operator in group $\mathrm{R}$ were similar with that in group P. There was also no significant difference between the two groups regarding the satisfaction of patients. Indeed, RT can provide not only a satisfactory operational state of the operator but also a comfortable anesthesia experience for patients. Therefore, it was assumed that RT was non-inferior to propofol in terms of its capacity as an intravenous agent used for general anesthesia. 
In a recent study, patients undergoing hysteroscopy were randomly divided into the RT group and propofol group. The incidence of adverse events during surgery, such as hypotension and bradycardia in group $\mathrm{R}$ was lower than that in group $\mathrm{P}^{27}$ Another study showed that the frequency of hypotension, low $\mathrm{SpO}_{2}$, and respiratory depression were lesser in group $\mathrm{R}$ than in group $\mathrm{P}$ in patients undergoing colonoscopy. ${ }^{28}$ Our study was conducted to investigate if cirrhotic patients undergoing EVL can obtain the benefits of RT compared with propofol. The RT group had a lower incidence of hypotension ( 2 [5.3\%]) versus 9 [23.7\%]) than the propofol group during surgery $(P<0.05)$. Post-operative low $\mathrm{SpO}_{2}$ event was not observed in group $\mathrm{R}(0[0 \%])$ compared to group $\mathrm{P}$ (6 [15.8\%], $P<0.05)$. Compared with group $\mathrm{R}$, the HR at $\mathrm{T} 1$ and T4-8 in group $\mathrm{P}$ were all significantly reduced $(P<0.05)$, but all values at T1-8 were within the clinically normal range. After anesthesia induction, the MAP at T18 in group $\mathrm{P}$ were significantly lower than that in group $\mathrm{R}(P<0.05)$. The fluctuation of MAP and HR in group $\mathrm{R}$ was lesser than that in group P. Our results were consistent with a previous study ${ }^{29}$ that vital signs can remain stable under RT sedation.

Our study has some limitations. First, since patients were not categorized according to the Child-Pugh scale, further studies are needed for evaluation. Second, this was a single center study with a limited sample size, and multicenter studies are required to further verify the conclusions of our study. However, the current study indeed demonstrated that patients undergoing EVL can benefit from RT general anesthesia.

\section{Conclusion}

Remimazolam tosilate general anesthesia and sequential flumazenil can provide satisfactory anesthetic effects and rapid recovery for cirrhotic patients undergoing EVL. Moreover, RT decreased the risk of adverse events, such as hypotension and low $\mathrm{SpO}_{2}$. $\mathrm{RT}$ is an effective option for general anesthesia, especially for cirrhotic patients.

\section{Data Sharing Statement}

The datasets are not publicly available due to the privacy policy. However, all authors in our team agree to share the datasets that generated during the present study after the article was published. The data can be obtained by log at http://www.chictr.org.cn after we upload the data.

\section{Author Contributions}

All authors made substantial contributions to the conception and design, acquisition of data, or analysis and interpretation of data; took part in drafting the article or revising it critically for important intellectual content; agreed to submit to the current journal; gave final approval of the version to be published; and agree to be accountable for all aspects of the work.

\section{Funding}

Special funds for comfortable medical anesthesia optimization of Shandong Provincial Medical Association, YXH2021ZX004.

\section{Disclosure}

All authors have no potential conflicts of interest to disclose.

\section{References}

1. Garcia-Tsao G, Bosch J. Management of varices and variceal hemorrhage in cirrhosis. $N$ Engl J Med. 2010;362:823-832. doi:10.1056/ NEJMra0901512

2. Mansour L, El-Kalla F, El-Bassat H, et al. Randomized controlled trial of scleroligation versus band ligation alone for eradication of gastroesophageal varices. Gastrointest Endosc. 2017;86:307-315. doi:10.1016/j.gie.2016.12.026

3. Alam L, Khattak MA, Alam M. Safety of balanced propofol and midazolam in upper gastrointestinal endoscopy for sedation in cirrhotic patients. J Pak Med Assoc. 2021;71:64-68. doi:10.47391/JPMA.526

4. Thuluvath PJ. Toward safer sedation in patients with cirrhosis: have we done enough? Gastrointest Endosc. 2009;70:269-271. doi:10.1016/j.gie.2009.01.029

5. Obara K, Haruma K, Irisawa A, et al. Guidelines for sedation in gastroenterological endoscopy. Dig Endosc. 2015;27:435-449. doi:10.1111/den. 12464

6. Reves JG, Samuelson PN, Vinik HR. Midazolam. Contemp Anesth Pract. 1983;7:147-162.

7. Hsu AJ, Carson KA, Yung R, Pham PA. Severe prolonged sedation associated with coadministration of protease inhibitors and intravenous midazolam during bronchoscopy. Pharmacotherapy. 2012;32:538-545. doi:10.1002/j.1875-9114.2011.01045.x

8. Vasudevan AE, Goh KL, Bulgiba AM. Impairment of psychomotor responses after conscious sedation in cirrhotic patients undergoing therapeutic upper GI endoscopy. $A m \quad J$ Gastroenterol. 2002;97:1717-1721. doi:10.1111/j.1572-0241.2002.05831.x

9. Wahab EA, Hamed EF, Ahmad HS, Abdel Monem SM, Fathy T. Conscious sedation using propofol versus midazolam in cirrhotic patients during upper GI endoscopy: a comparative study. JGH Open. 2019;3:25-31. doi:10.1002/jgh3.12098

10. Starczewska MH, Mon W, Shirley P. Anaesthesia in patients with liver disease. Curr Opin Anaesthesiol. 2017;30:392-398. doi:10.1097/ACO.0000000000000470

11. Ziser A, Plevak DJ. Morbidity and mortality in cirrhotic patients undergoing anesthesia and surgery. Curr Opin Anaesthesiol. 2001;14:707-711. doi:10.1097/00001503-200112000-00018

12. Tang L, Liu H, Wu Y, et al. Sevoflurane may be more beneficial than propofol in patients receiving endoscopic variceal ligation and endoscopic variceal sclerotherapy: a randomized, double-blind study. Exp Ther Med. 2017;14:3145-3152. doi:10.3892/etm.2017.4919 
13. Zhou Y, Hu P, Huang Y, et al. Population Pharmacokinetic/ Pharmacodynamic model-guided dosing optimization of a novel sedative HR7056 in Chinese healthy subjects. Front Pharmacol. 2018;9:1316. doi:10.3389/fphar.2018.01316

14. Chen SH, Yuan TM, Zhang J, et al. Remimazolam tosilate in upper gastrointestinal endoscopy: a multicenter, randomized, non-inferiority, Phase III trial. $J$ Gastroenterol Hepatol. 2021;36:474-481. doi:10.1111/jgh.15188

15. Lin BF, Huang YS, Kuo CP, et al. Comparison of A-line autoregressive index and observer assessment of alertness/sedation scale for monitored anesthesia care with target-controlled infusion of propofol in patients undergoing percutaneous vertebroplasty. J Neurosurg Anesthesiol. 2011;23:6-11. doi:10.1097/ANA.0b013e3181ecbdbf

16. Jo HB, Lee JK, Jang DK, et al. Safety and effectiveness of midazolam for cirrhotic patients undergoing endoscopic variceal ligation. Turk J Gastroenterol. 2018;29:448-455. doi:10.5152/tjg.2018.17589

17. Unal HU, Saruc M. Is midazolam safe for sedation in cirrhotic patients? Turk J Gastroenterol. 2020;31:75. doi:10.5152/tjg.20 19.18864

18. Mao W, Wei XQ, Tao J, Zhen FP, Wen ZF, Wu B. The safety of combined sedation with propofol plus fentanyl for endoscopy screening and endoscopic variceal ligation in cirrhotic patients. J Dig Dis. 2014;15:124-130. doi:10.1111/1751-2980.12115

19. Sethi S, Wadhwa V, Thaker A, et al. Propofol versus traditional sedative agents for advanced endoscopic procedures: a meta-analysis. Dig Endosc. 2014;26:515-524. doi:10.1111/den.12219

20. Watanabe K, Hikichi T, Takagi T, et al. Propofol is a more effective and safer sedative agent than midazolam in endoscopic injection sclerotherapy for esophageal varices in patients with liver cirrhosis: a randomized controlled trial. Fukushima $J$ Med Sci. 2018;64:133-141. doi:10.5387/fms.2018-21

21. Chen X, Yan R, Bai Z, Ma H. Enhanced sedative efficacy and delayed recovery in propofol anesthesia in a rat model of hepatic cirrhosis. Int J Clin Exp Med. 2015;8:5723-5730.
22. Schuttler J, Eisenried A, Lerch M, Fechner J, Jeleazcov C, Ihmsen H Pharmacokinetics and pharmacodynamics of remimazolam (CNS 7056) after continuous infusion in healthy male volunteers: part I. Pharmacokinetics and clinical pharmacodynamics. Anesthesiology. 2020;132:636-651. doi:10.1097/ALN.0000000000003103

23. Stohr T, Colin PJ, Ossig J, et al. Pharmacokinetic properties of remimazolam in subjects with hepatic or renal impairment. $\mathrm{Br}$ J Anaesth. 2021;127:415-423. doi:10.1016/j.bja.2021.05.027

24. Chen X, Sang N, Song K, et al. Psychomotor recovery following remimazolam-induced sedation and the effectiveness of flumazenil as an antidote. Clin Ther. 2020;42:614-624. doi:10.1016/j.clinthera.20 20.02.006

25. Doi M, Morita K, Takeda J, Sakamoto A, Yamakage M, Suzuki T. Efficacy and safety of remimazolam versus propofol for general anesthesia: a multicenter, single-blind, randomized, parallel-group, phase IIb/III trial. J Anesth. 2020;34:543-553. doi:10.1007/s00540020-02788-6

26. Johansen JW. Update on bispectral index monitoring. Best Pract Res Clin Anaesthesiol. 2006;20:81-99. doi:10.1016/j.bpa.2005.08.004

27. Zhang X, Li S, Liu J. Efficacy and safety of remimazolam besylate versus propofol during hysteroscopy: single-centre randomized controlled trial. BMC Anesthesiol. 2021;21:156. doi:10.1186/s12871021-01373-y

28. Chen $\mathrm{S}$, Wang J, Xu X, et al. The efficacy and safety of remimazolam tosylate versus propofol in patients undergoing colonoscopy: a multicentered, randomized, positive-controlled, phase III clinical trial. Am J Transl Res. 2020;12:4594-4603.

29. Pastis NJ, Yarmus LB, Schippers F, et al. Safety and efficacy of remimazolam compared with placebo and midazolam for moderate sedation during bronchoscopy. Chest. 2019;155:137-146. doi:10.1016/j.chest.2018.09.015
International Journal of General Medicine

\section{Publish your work in this journal}

The International Journal of General Medicine is an international, peer-reviewed open-access journal that focuses on general and internal medicine, pathogenesis, epidemiology, diagnosis, monitoring and treatment protocols. The journal is characterized by the rapid reporting of reviews, original research and clinical studies across all disease areas. The manuscript management system is completely online and includes a very quick and fair peer-review system, which is all easy to use. Visit http://www.dovepress.com/ testimonials.php to read real quotes from published authors. 